\title{
A theory of qualified types
}

\author{
Mark P. Jones \\ Programming Research Group \\ Oxford University Computing Laboratory \\ 8-11 Keble Road \\ Oxford OX1 3QD \\ (email: mpj@prg.ox.ac.uk)
}

\section{Introduction}

In a language with a polymorphic type systern, a term of type $\forall t . f(t)$ can be treated (possibly after suitable instantiation) as having any of the types in the set:

$$
\{f(t) \mid t \text { is a type }\} .
$$

It is natural to consider a more restricted form of polymorphism in which the value taken by $t$ may be constrained to a particular subset of types. In this situation, we write $\forall t . \pi(t) \Rightarrow f(t)$, where $\pi(t)$ is a predicate on types, for the type of an object which can be treated (after suitable instantiation) as having any of the types in the set:

$$
\{f(t) \mid t \text { is a type such that } \pi(t) \text { holds }\} .
$$

A term with a restricted polymorphic type of this kind is often said to be overloaded, having different interpretations for different argument types.

This paper presents a general theory of overloading based on the use of qualified types, which are types of the form $\pi \Rightarrow \sigma$ denoting those instances of type $\sigma$ which satisfy the predicate $\pi$. The main benefits of using qualified types are:

- A general approach which includes a range of familiar type systems as special cases. Results and tools developed for the general system are immediately applicable to each particular application.

- A precise treatment of the relationship between implicit and explicit overloading. This is particularly useful for describing the implementation of systems supporting qualified types.

- The ability to include local constraints as part of the type of an object. This enables the definition and use of polymorphic overloaded values within a program.

\section{Outline of paper}

Each of the type systems considered in this paper is parameterised by the choice of a system of predicates on type expressions, whose basic properties are described in Section 1. A number of 
examples are included to illustrate the use of this framework to describe a range of type systems including Haskell type classes, extensible records and subtyping. Section 2 describes the use of qualified types in the context of polymorphic $\lambda$-calculus with explicit typing. This is extended in Section 3 using a general notion of evidence to explore the relationship between implicit and explicit overloading. An alternative approach, suitable for use in an implicitly typed language, is introduced in Section 4 using an extension of the ML type system [Mil78] to support qualified types. Although substantially less powerful than polymorphic $\lambda$-calculus, we show that the resulting system is suitable for use in a language based on type inference, which allows the type of a term to be determined without explicit type annotations.

\section{Predicates}

Each of the type systems considered in this paper is parameterised by the choice of a language of predicates $\pi$ together with an entailment relation th between (finite) sets of predicates. Individual predicates may be written using expressions of the form $\pi=p \tau_{1} \ldots \tau_{n}$ where $p$ is a predicate symbol corresponding to an $n$-place relation between types; the predicate $\pi$ represents the assertion that the types $\tau_{1}, \ldots, \tau_{n}$ are in this relation. The definition of $\mathbb{H}$ varies from one application to another. The only properties that we will assume are:

- monotonicity. $P H P^{\prime}$ whenever $P \supseteq P^{\prime}$.

- transitivity. if $P H Q$ and $Q H R$ then $P H R$.

- closure property. if $P H Q$ then $S P H S Q$ for any substitution $S$ mapping type variables (and hence type expressions) to type expressions.

If $P$ is a set of predicates and $\pi$ is a predicate then we write $P H \pi$ and $P, \pi$ as abbreviations for $P H-\{\pi\}$ and $P \cup\{\pi\}$ respectively.

The following subsections illustrate the languages of predicates used in three applications of qualified types. Only the basic ideas are sketched here; further details may be found in [Jon91a].

\subsection{Example: type classes}

Introduced in [WB89] and adopted as part of the standard for the programming language Haskell [HPJW91], type classes are particularly useful for describing the implementation of standard polymorphic operators such as computable equality. Much of the original motivation for qualified types came from the study of type classes.

Broadly speaking, a type class is a family of types (the instances of the class) on which a number of values (the member functions) are defined. In this case, each predicate symbol corresponds to a user-defined class and a predicate of the form $C \tau$ represents the assertion that the type $\tau$ is an instance of the class named $C$. The class $E q$ is a standard example whose instances are those types whose elements can be tested for equality using the operator $(==): \forall a . E q a \Rightarrow a \rightarrow a \rightarrow B o o l$.

Differences in the basic approach to type classes are reflected in the properties of the H- relation. In a standard Haskell system we have axioms such as $\emptyset \Vdash E q$ Int and $E q$ a $\# E q[a]$. The same 
framework can also be used to describe the use of Haskell superclasses, and to support the extension to classes with multiple parameters as used in Gofer [Jon92].

Type classes are best suited to systems with a type inference algorithm such as that described in Section 6 where the appropriate instances of each overloaded operator can be determined automatically as part of the type inference process.

\subsection{Example: extensible records}

A record is a set of values labelled by the elements $l$ of a specified set of labels. There has been considerable interest in the use of record types to model inheritance in object oriented programming languages and a number of different approaches have been considered. Using the type system to be described in Section 2 we can construct a system of extensible records, strongly reminiscent of [HP90] using predicates of the form:

$r$ has $l: t \quad$ indicating that a record of type $r$ has a field labelled $l$ of type $t$.

$r$ lacks $l \quad$ indicating that a record of type $r$ does not have a field labelled $l$.

This also requires an extension of the language of type expressions to allow types of the form $\langle$ (the empty record, which lacks any fields), $r \backslash l$ (the type of a record obtained by removing a field labelled $l$ from a record of type $r$ ) and $(r \mid l: t)$ (the type of a record obtained by extending a record of type $r$ with a new field of type $t$ labelled $l)$. The definition of the entailment relation includes axioms such as $\emptyset H(\langle\rangle$ lacks $l)$ and $\{r$ lacks $l\} \nVdash((r \mid l: t)$ has $l: t)$.

The primitive operations of record restriction, extension and selection can then be represented by families of functions (indexed by labels) of type:

$$
\begin{array}{ll}
(-\backslash l) & :: \forall r . \forall t .(r \text { has } l: t) \Rightarrow r \rightarrow r \backslash l \\
(-\mid l=-) & :: \forall r . \forall t .(r \text { lacks } l) \Rightarrow r \rightarrow t \rightarrow(r \mid l: t) \\
(-. l) & :: \forall r . \forall t .(r \text { has } l: t) \Rightarrow r \rightarrow t
\end{array}
$$

Details of the relationship between this approach and those of [Rem89, CM90] are given in [HP90].

\subsection{Example: subtyping}

Type systems with various forms of subtyping can be described using predicates of the form $\sigma \subseteq$. $\sigma^{\prime}$, representing the assertion that $\sigma$ is a subtype of $\sigma^{\prime}$. Many such systems, including those of [Mit84, FM89], allow the use of implicit coercion from one type to another. The extensions required to support this are discussed in Section 7.3.

\section{Polymorphic $\lambda$-calculus with qualified types}

\subsection{Basic definitions}

In this section, we work with a variant of polymorphic $\lambda$-calculus which includes qualified types using type expressions of the form:

$$
\sigma::=t|\sigma \rightarrow \sigma| \forall t . \sigma \mid \pi \Rightarrow \sigma
$$


where $t$ ranges over a given set of type variables. The $\rightarrow$ and $\Rightarrow$ symbols are treated as right associative infix binary operators with $\rightarrow$ binding more tightly than $\Rightarrow$. Additional type constructors such as those for integers, lists and record types will be used as required. The set of type variables appearing (free) in an expression $X$ is denoted $T V(X)$.

To begin with we use an unmodified form of the (unchecked) terms of polymorphic $\lambda$-calculus, given by expressions of the form:

$$
M::=x|M N| \lambda x: \sigma . M|M \sigma| \lambda t . M
$$

where $x$ ranges over a given set of term variables. The set of free (term) variables appearing in a term $M$ will be denoted $F V(M)$. Note that we do not provide constructs for the introduction of new overloadings such as inst and over in [WB89]. If none of the free variables for a given term have qualified (i.e. overloaded) types then no overloading will be used in the expression.

\subsection{Typing rules}

A type assignment is a (finite) set of typing statements of the form $x: \sigma$ in which no term variable $x$ appears more than once. If $A$ is a type assignment, then we write $\operatorname{dom} A=\{x \mid(x: \sigma) \in A\}$, and if $x$ is a term variable with $x \notin \operatorname{dom} A$ then we write $A, x: \sigma$ as an abbreviation for the type assignment $A \cup\{x: \sigma\}$. The type assignment obtained from $A$ by removing any typing statement for the variable $x$ is denoted $A_{x}$. A type assignment $A$ can be interpreted as a function mapping each element of $\operatorname{dom} A$ to a type scheme. In particular, if $(x: \sigma) \in A$ then we write $A(x)=\sigma$.

An expression of the form $P \mid A \vdash M: \sigma$ represents the assertion that the term $M$ has type $\sigma$ when the predicates in $P$ are satisfied and the types of free variables in $M$ are as specified in the type assignment $A$. The typing rules for this system are given in Figure 1. Most of these are similar to the rules for explicit typing of polymorphic $\lambda$-calculus and do not involve the predicate set.

\begin{tabular}{|ccc|}
\hline Standard rules: & $($ var $)$ & $P \mid A \vdash x: \sigma$
\end{tabular}$\quad(x: \sigma) \in A$

Figure 1: Typing rules for polymorphic $\lambda$-calculus with qualified types 
By an abuse of notation, we will also use $P \mid A \vdash M: \sigma$ as a proposition asserting the existence of a derivation of $P \mid A \vdash M: \sigma$.

\section{Evidence}

Although the system of qualified types described in the previous sections is suitable for reasoning about the types of overloaded terms, it cannot be used to describe their evaluation. For example, the knowledge that $I n t$ is an instance of the class $E q$ is not sufficient to determine the value of the expression $2==3$; we must also be provided with the value of the equality operator which makes Int an instance of $E q$. In general, we can only use a term of type $\pi \Rightarrow \sigma$ if we are also supplied with suitable evidence that the predicate $\pi$ does indeed hold.

This leads us to consider an extension of the term language which makes the role of evidence explicit, using:

- Evidence expressions: A language of evidence expressions e denoting evidence values, including a set of evidence variables $v$.

- Evidence construction: An evidence assignment is a set of elements of the form $(v: \pi)$ in which no evidence variable appears more than once. The H- relation is extended to a three place relation $P H e: \pi$, indicating that it is possible to construct evidence $e$ for the predicate $\pi$ in any environment binding the variables in the evidence assignment $P$ to appropriate evidence values. Thus predicates play a similar role for evidence expressions as types for simple $\lambda$-calculus terms.

- Evidence abstraction: A term $M$ of type $\pi \Rightarrow \rho$ is implemented by a term of the form $\lambda v: \pi . M^{\prime}$ where $v$ is an evidence variable and $M^{\prime}$ is a term of type $\rho$ corresponding to $M$ using $v$ in each place where evidence for $\pi$ is needed.

- Evidence application: Each use of an overloaded expression $N$ of type $\pi \Rightarrow \rho$ is replaced by a term of the form $N^{\prime} e$ where $N^{\prime}$ is a term corresponding to $N$ and $e$ is an evidence expression for $\pi$.

- Evidence reduction: The standard rules of computation are augmented by a variant of $\beta$-reduction for evidence abstraction and application:

$$
(\lambda v . M) e \triangleright_{\beta e}[e / v] M .
$$

Most of the typing rules given in Figure 1 can be used with the extended system without modification. The only exceptions are the rules for dealing with qualified types; suitably modified versions of these are given in Figure 2.

Notice that extending the term language to make the use of evidence explicit gives unicity of type; each well-typed term has a uniquely determined type. This approach is very similar to the technique used to make polymorphism explicit in the translation from implicit to explicit typed $\lambda$-calculus using abstraction and application over types [Mit90]. As in that situation, there is a simple correspondence 


$$
\begin{aligned}
& (\Rightarrow E) \quad \frac{P, v: \pi \mid A \vdash M: \sigma}{P \mid A \vdash \lambda v: \pi \cdot M: \pi \Rightarrow \sigma} \\
& (\Rightarrow I) \quad \frac{P \mid A \vdash M: \pi \Rightarrow \sigma \quad P \Vdash e: \pi}{P \mid A \vdash M e: \sigma}
\end{aligned}
$$

Figure 2: Modified rules for qualified types with evidence

between derivations in the two systems, described by means of a function Erase mapping explicitly overloaded terms to their implicitly overloaded counterparts:

$$
\begin{array}{ll}
\operatorname{Erase}(x) & =x \\
\operatorname{Erase}(M N) & =(\operatorname{Erase}(M))(\operatorname{Erase}(N)) \\
& \vdots \\
\operatorname{Erase}(\lambda v: \pi \cdot M) & =\operatorname{Erase}(M) \\
\operatorname{Erase}(M e) & =\operatorname{Erase}(M)
\end{array}
$$

The correspondence between the two systems can now be described by:

Theorem $1 P \mid A \vdash M: \sigma$ using the original typing rules if and only if $P^{\prime} \mid A \vdash M^{\prime}: \sigma$ by a derivation of the same structure in the extended system such that $P=\left\{\pi \mid(v: \pi) \in P^{\prime}\right\}$ and $\operatorname{Erase}\left(M^{\prime}\right)=M$.

Given a term $M$ in the original system, each corresponding term using explicit overloading is called a translation (or implementation) of $M$ and can be used to give a semantics for the term. We use the notation $P^{\prime} \mid A \vdash M \sim M^{\prime}: \sigma$ to refer to the translation of a term in a specific context. Note that the translation of a given term may not be uniquely defined (with distinct translations corresponding to distinct derivations of $P \mid A \vdash M: \sigma$ ). This problem is discussed in more detail in Section 7.1.

The form of evidence required will vary from one application to another. Suitable choices for each of the examples described in Section 1 are as follows:

- Type classes: The evidence for a type class predicate of the form $C r$ is a dictionary (i.e. a tuple or record) containing the values of the members of $C$ at the instance $\tau$.

- Extensible records: The evidence for a predicate of the form ( $r$ lacks $l$ ) is the function:

$$
\left(-\mid l={ }_{-}\right):: \forall t . r \rightarrow t \rightarrow(r \mid l: t)
$$

The evidence for a predicate of the form $(r$ has $l: t)$ is the pair of functions:

$$
\begin{aligned}
& (-\backslash l):: r \rightarrow r \backslash l \\
& (-. l) \quad:: r \rightarrow t
\end{aligned}
$$

In practice, a concrete implementation of extensible records is likely to use offsets into a table of values used to store a record as evidence, passing these values to generic functions for updating or selecting from a record where necessary.

- Subtypes: The evidence for a predicate $\sigma \subseteq \sigma^{\prime}$ is a coercion function which maps values of type $\sigma$ to values of type $\sigma^{\prime}$. 


\section{An extension of ML using qualified types}

Polymorphic $\lambda$-calculus is not a suitable language to describe an implicitly typed language in which the need for explicit type annotations is replaced by the existence of a type inference algorithm. In practice, the benefits of type inference are often considered to outweigh the disadvantages of a less powerful type system. The ML type system [Mil78, DM82] is a well-known example in which the price of type inference is the inability to define functions with polymorphic arguments. Nevertheless, the ML type system has proved to be very useful in practice and has been adopted by a number of later languages.

Detailed proofs of the results presented in this and following sections may be found in [Jon91b]; they are not included here for reasons of space.

\subsection{Basic definitions}

Following the definition of types and type schemes in $M L$ we consider a structured language of types, with the principal restriction being the inability to support functions with either polymorphic or overloaded arguments:

$$
\begin{array}{lll}
\tau::=t \mid \tau \rightarrow \tau & \text { types } \\
\rho::=P \Rightarrow \tau & \text { qualified types } \\
\sigma::=\forall T . \rho & \text { type schemes }
\end{array}
$$

( $P$ and $T$ range over finite sets of predicates and finite sets of type variables respectively).

It is convenient to introduce some abbreviations for qualified type and type scheme expressions. In particular, if $\rho=(P \Rightarrow \tau)$ and $\sigma=\forall T$. $\rho$ then we write:

\begin{tabular}{|cc|cc|}
\hline Abbreviation & Qualified type & Abbreviation & Type scheme \\
\hline$\tau$ & $\emptyset \Rightarrow \tau$ & $\rho$ & $\forall \emptyset . \rho$ \\
$\pi \Rightarrow \rho$ & $P, \pi \Rightarrow \tau$ & $\forall t . \sigma$ & $\forall(T \cup\{t\}) . \rho$ \\
$P^{\prime} \Rightarrow \rho$ & $P \cup P^{\prime} \Rightarrow \tau$ & $\forall T^{\prime} . \sigma$ & $\forall\left(T \cup T^{\prime}\right) . \rho$ \\
\hline
\end{tabular}

In addition, if $\left\{\alpha_{i}\right\}$ is an indexed set of variables, we write $\forall \alpha_{i} . \rho$ as an abbreviation for $\forall\left\{\alpha_{i}\right\} . \rho$. As usual, type schemes are regarded as equal if they are equivalent upto renaming of bound variables. Using this notation, any type scheme can be written in the form $\forall \alpha_{i} . P \Rightarrow \tau$, representing the set of qualified types:

$$
\left\{\left[\tau_{i} / \alpha_{i}\right] P \Rightarrow\left[\tau_{i} / \alpha_{i}\right] \tau \mid \tau_{i} \in T y p e\right\}
$$

where $\left[\tau_{i} / \alpha_{i}\right]$ is the substitution mapping each of the variables $\alpha_{i}$ to the corresponding type $\tau_{i}$ and Type is the set of all simple type expressions.

As in [Mil78, DM82, CDK86], we use a term language based on simple untyped $\lambda$-calculus with the addition of a let construct to enable the definition and use of polymorphic (and in this case, overloaded) terms.

$$
M::=x|M N| \lambda x . M \mid \text { let } x=M \text { in } N
$$

A suitable set of typing rules for this system is given in Figure 3. Note the use of the symbols $\tau, \rho$ and $\sigma$ to restrict the application of certain rules to specific sets of type expressions. 


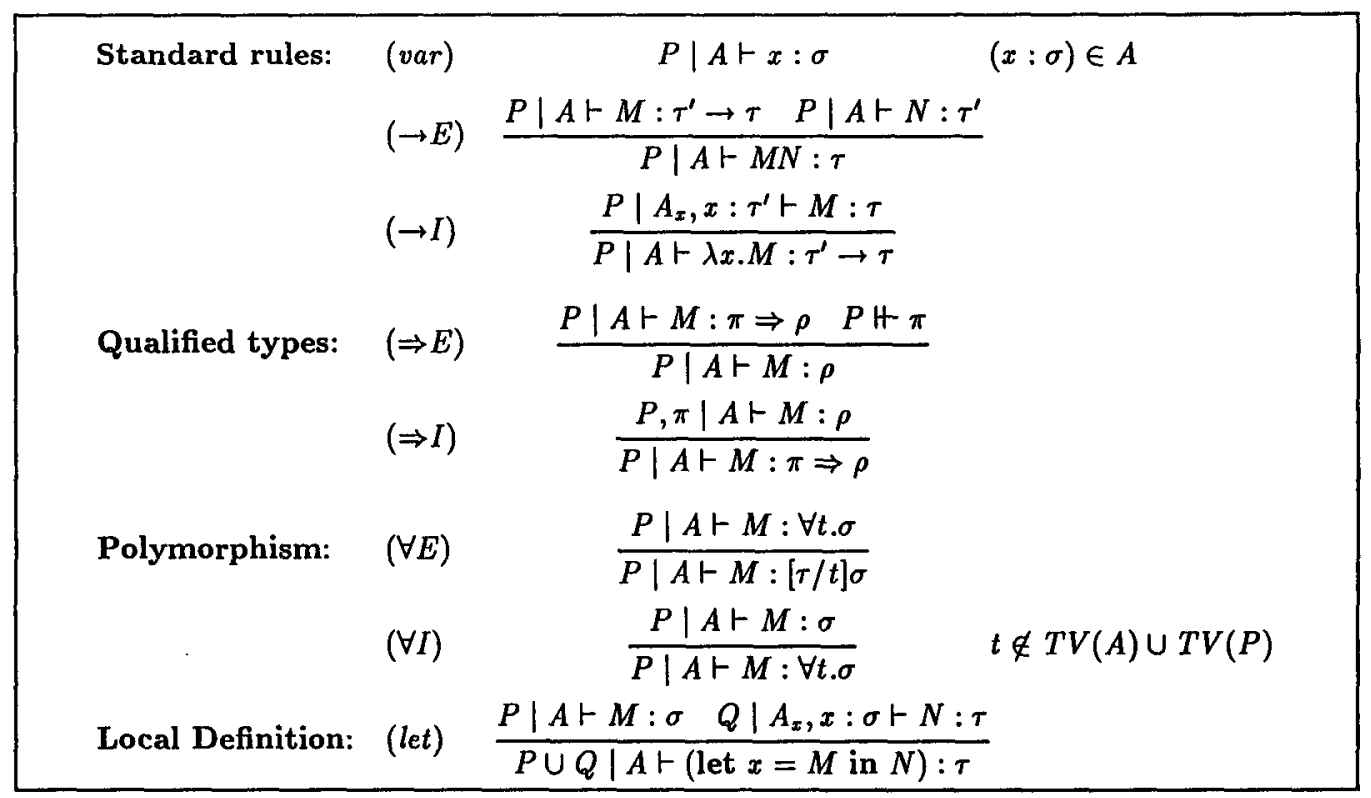

Figure 3: ML-like typing rules for qualified types

\subsection{Constrained type schemes}

A typing judgement $P \mid A \vdash M: \sigma$ assigns a type scheme $\sigma$ to a term $M$, but also constrains uses of this typing to environments satisfying the predicates in $P$. It is therefore convenient to introduce a notation for a type scheme coupled with a set of predicates specifying constraints on the environment in which the type scheme may be used.

Definition $1 A$ constrained type scheme is an expression of the form $(P \mid \sigma)$ where $P$ is a set of predicates and $\sigma$ is a type scheme.

Note that a type scheme $\sigma$ may be treated as an abbreviation for the constrained type scheme $(\theta \mid \sigma)$ (and in fact, every constrained type scheme can be represented by a simple type scheme using a renaming of bound variables).

Definition $2 A$ qualified type $R \Rightarrow \mu$ is said to be a generic instance of the constrained type scheme $\left(P \mid \forall \alpha_{i} \cdot Q \Rightarrow \tau\right)$ if there are types $\tau_{i}$ such that:

$$
R H P \cup\left[\tau_{i} / \alpha_{i}\right] Q \text { and } \mu=\left[\tau_{i} / \alpha_{i}\right] \tau .
$$

The generic instance relation can be used to define a general ordering $(\leq)$ on constrained type schemes. The principal motivation for the definition of this relation is that a statement of the form $\sigma^{\prime} \leq \sigma$ should mean that it is possible to use an object of type $\sigma$ wherever an object of type $\sigma^{\prime}$ is required.

Definition 3 The constrained type scheme $(Q \mid \eta)$ is said to be more general than a constrained type scheme $(P \mid \sigma)$, written $(P \mid \sigma) \leq(Q \mid \eta)$, if $\rho$ is a generic instance of $(P \mid \sigma)$ whenever it is a generic instance of $(Q \mid \eta)$. 
It is straightforward to show that this defines a preorder on the set of constrained type schemes, such that a qualified type $\rho$ is a generic instance of the type scheme $\sigma$ if and only if $\rho \leq \sigma$. Although ( $\leq$ ) is far from anti-symmetric, we will usually treat it as such by regarding type schemes as equal if they are equivalent under $(\leq)$. The following properties are easily established:

- If $\rho$ is a qualified type and $P$ is a set of predicates, then $(P \mid \rho)=P \Rightarrow \rho$.

- If $\sigma$ is a type scheme and $P$ is a set of predicates then $(P \mid \sigma) \leq \sigma$.

- If $\sigma^{\prime} \leq \sigma$ and $P^{\prime} H P$ then $\left(P^{\prime} \mid \sigma^{\prime}\right) \leq(P \mid \sigma)$.

- If none of the variables $\alpha_{i}$ appear in $P$, then the constrained type scheme $\left(P \mid \forall \alpha_{i} . \rho\right)$ is equivalent to the type scheme $\forall \alpha_{i} . P \Rightarrow \rho$.

The application of a substitution $S$ to a constrained type scheme $(P \mid \sigma)$ is defined by $S(P \mid \sigma)=$ $(S P \mid S \sigma)$. The next proposition describes an important property of the ordering on constrained type schemes.

Proposition 1 For any substitution $S$ and constrained type schemes $(P \mid \sigma)$ and $(Q \mid \eta)$ :

$$
(P \mid \sigma) \leq(Q \mid \eta) \Rightarrow S(P \mid \sigma) \leq S(Q \mid \eta)
$$

\subsection{Ordering of type assignments}

The definition of constrained type schemes and the ordering $(\leq)$ extends naturally to an ordering on (constrained) type assignments.

Definition 4 If $A$ and $A^{\prime}$ are type assignments and $P, P^{\prime}$ are sets of predicates, then we say that $(P \mid A)$ is more general than $\left(P^{\prime} \mid A^{\prime}\right)$, written $\left(P^{\prime} \mid A^{\prime}\right) \leq(P \mid A)$, if dom $A=\operatorname{dom} A^{\prime}$ and $\left(P^{\prime} \mid A^{\prime}(x)\right) \leq(P \mid A(x))$ for each $x \in \operatorname{dom} A$.

The results of the previous section can be used to prove that this ordering on type assignments is reflexive, transitive and preserved by substitutions. In this paper, we will only use the special case where $P=\emptyset$ in which case we write $\left(P^{\prime} \mid A^{\prime}\right) \leq A$. This can be interpreted as indicating that each of the types assigned to a variable in $A$ is more general than the type assigned in $A^{\prime}$ in any environment which satisfies the predicates in $P^{\prime}$.

\subsection{Generalisation}

Given a derivation $P \mid A \vdash M: \tau$, it is useful to have a notation for the most general type scheme that can be obtained for $M$ from this derivation using the rules $(\Rightarrow I)$ and $(\forall I)$ given in Figure 3.

Definition 5 The generalisation of a qualified type $\rho$ with respect to a type assignment $A$ is written $\operatorname{Gen}(A, \rho)$ and defined by:

$$
G e n(A, \rho)=\forall(T V(\rho) \backslash T V(A)) . \rho .
$$


In other words, if $\left\{\alpha_{i}\right\}=T V(\rho) \backslash T V(A)$, then $G e n(A, \rho)=\forall \alpha_{i} . \rho$. The following propositions describe the interaction of generalisation with predicate entailment and substitution.

Proposition 2 Suppose that $A$ is a type assignment, $P$ and $P^{\prime}$ are sets of predicates and $\tau$ is a type. Then $\operatorname{Gen}\left(A, P^{\prime} \Rightarrow \tau\right) \leq \operatorname{Gen}(A, P \Rightarrow \tau)$ whenever $P^{\prime}+P$.

Proposition 3 If $A$ is a type assignment, $\rho$ is a qualified type and $S$ is a substitution then:

$$
\operatorname{Gen}(S A, S \rho) \leq S(\operatorname{Gen}(A, \rho)) .
$$

Furthermore, there is a substitution $R$ such that:

$$
R A=S A \text { and } \operatorname{SGen}(A, \rho)=\operatorname{Gen}(R A, R \rho) .
$$

\section{A syntax-directed approach}

The typing rules in Figure 3 provide clear descriptions of the treatment of each of the syntactic constructs of the term and type languages. Unfortunately, they are not suitable for use in a type inference algorithm where it should be possible to determine an appropriate order in which to apply the typing rules by a simple analysis of the syntactic structure of the term whose type is required.

In this section, we introduce an alternative set of typing rules with a single rule for each syntactic construct in the term language. We refer to this as the syntax-directed system because it has the following important property:

All typing derivations for a given term $M$ (if there are any) have the same structure, uniquely
determined by the syntactic structure of $M$.

We regard the syntax-directed system as a tool for exploring the type system of Section 4 and we establish a congruence between the two systems so that results about one can be translated into results about the other. The advantages of working with the syntax-directed system are:

- The rules are better suited to use in a type inference algorithm; having found types for each of the subterms of a given term $M$, there is at most one rule which can be used to obtain a type for the term $M$ itself.

- Only type expressions are involved in the matching process. Type schemes and qualified types can only appear in type assignments.

- There are fewer rules and hence fewer cases to be considered in formal proofs.

A similar approach is described in [CDK86] which gives a deterministic set of typing rules for ML and outlines their equivalence to the rules in [DM82].

\subsection{Syntax-directed typing rules}

The typing rules for the syntax-directed system are given in Figure 4. Typings in this system are written in form $P \mid A \stackrel{s}{\vdash} M: \tau$, where $\tau$ ranges over the set of type expressions rather than the set of type schemes as in the typing judgements of Section 4. Other than this, the principal differences between the two systems are in the rules $(v a r)^{s}$ and $(\text { let })^{s}$ which use the operations of instantiation and generalisation introduced in Sections 4.2 and 4.4 . 


$$
\begin{aligned}
& (\text { var })^{*} \quad \frac{(x: \sigma) \in A}{P \mid A \vdash x: \tau} \quad(P \Rightarrow \tau) \leq \sigma \\
& (\rightarrow E)^{s} \frac{P\left|A \dot{\vdash} M: \tau^{\prime} \rightarrow \tau \quad P\right| A \dot{\vdash} N: \tau^{\prime}}{P \mid A \dot{\vdash} M N: \tau} \\
& (\rightarrow I)^{s} \quad \frac{P \mid A_{x}, x: \tau^{\prime} \stackrel{\vdash}{ } M: \tau}{P \mid A \dot{\vdash} \lambda x . M: \tau^{\prime} \rightarrow \tau} \\
& \frac{P\left|A \stackrel{\vdash}{\vdash}: \tau \quad P^{\prime}\right| A_{x}, x: \sigma \stackrel{\vdash}{ } N: \tau^{\prime}}{P^{\prime} \mid A \vdash^{\prime}(\operatorname{let} x=M \text { in } N): \tau^{\prime}} \quad \sigma=\operatorname{Gen}(A, P \Rightarrow \tau)
\end{aligned}
$$

Figure 4: Syntax-directed inference system

\subsection{Properties of the syntax-directed system}

The following proposition illustrates the parametric polymorphism present in the syntax-directed system; instantiating the free type variables in a derivable typing with arbitrary types produces another derivable typing.

Proposition 4 If $P \mid A \dot{\vdash} M: \tau$ and $S$ is a substitution then $S P \mid S A \dot{\vdash} M: S \tau$.

A similar result is established in [Dam85] where it is shown that for any derivation $A \vdash M: \tau$ in the usual (non-deterministic) ML type system and any substitution $S$, there is a derivation $S A \vdash M: S \tau$ which can be chosen in such a way that the height of the latter is bounded by the height of the former. This additional condition is needed to ensure the validity of proofs by induction on the size of a derivation. This complication is avoided by the syntax-directed system; the derivations in proposition 4 are guaranteed to have the same structure because the term $M$ is common to both.

Proposition 5 If $P \mid A \stackrel{\bullet}{\vdash}: \tau$ and $Q H P$ then $Q \mid A \dot{\vdash} M: \tau$.

This result describes a form of polymorphism over the sets of environments in which a particular typing can be used. This seemingly obvious property does not hold in the original type system; it is possible for $Q$ to contain variables not mentioned in $P$ and hence to prohibit the use of $(\forall I)$ where it might have otherwise been applicable.

Proposition 6 If $P \mid A^{\prime} \dot{\vdash} M: \tau$ and $\left(P \mid A^{\prime}\right) \leq A$ then $P \mid A \dot{\leftarrow} M: \tau$.

The hypothesis $\left(P \mid A^{\prime}\right) \leq A$ means that the types assigned to variables in $A$ are more general than those given by $A^{\prime}$ in any environment which satisfies the predicates in $P$. For example:

$$
(E q \operatorname{Int} \mid\{(==): \operatorname{Int} \rightarrow \operatorname{Int} \rightarrow \text { Bool }\}) \leq\{(==): \forall a . E q a \Rightarrow a \rightarrow a \rightarrow \text { Bool }\}
$$

and hence, by the proposition above, it is possible to replace an integer equality function with a generic equality function of type $\forall a . E q a \Rightarrow a \rightarrow a \rightarrow B o o l$ in any environment which satisfies Eq Int. 


\subsection{Relationship with original type system}

In order to use the syntax-directed system as a tool for reasoning about the type system described in Section 4, we need to investigate the way in which the existence of a derivation in one system determines the existence of derivations in the other.

Our first result establishes the soundness of the syntax-directed system with respect to the original typing rules, showing that any derivable typing in the former system is also derivable in the latter.

Theorem 2 If $P \mid A \stackrel{\leftrightarrow}{\vdash} M: \tau$ then $P \mid A \vdash M: \tau$.

The translation of derivations in the original type system to those of the syntax-directed system is less obvious. For example, if $P \mid A \vdash M: \sigma$ then it will not in general be possible to derive the same typing in the syntax-directed system because $\sigma$ is a type scheme, not a simple type. However, for any derivation $P^{\prime} \mid A \stackrel{s}{\vdash} M: \tau$, theorem 2 guarantees the existence of a derivation $P^{\prime} \mid A \vdash M: \tau$ and hence $\emptyset \mid A \vdash M: \operatorname{Gen}\left(A, P^{\prime} \Rightarrow \tau^{\prime}\right)$ by definition 5. The following theorem shows that it is always possible to find a derivation in this way such that the inferred type scheme $\operatorname{Gen}\left(A, P^{\prime} \Rightarrow \tau^{\prime}\right)$ is more general than the constrained type scheme $(P \mid \sigma)$ determined by the original derivation.

Theorem 3 If $P \mid A \vdash M: \sigma$ then there is a set of predicates $P^{\prime}$ and a type $\tau$ such that $P^{\prime} \mid A \dot{\vdash} M: \tau$ and $(P \mid \sigma) \leq \operatorname{Gen}\left(A, P^{\prime} \Rightarrow \tau\right)$.

\section{A type inference algorithm}

In this section, we give an algorithm for calculating a typing for a given term, using an extension of Milners algorithm $W$ [Mil78] to support qualified types. We show that the typings produced by this algorithm are derivable in the syntax-directed system and that they are, in a certain sense, the most general typings possible. Combining this with the results of the previous section, the algorithm can be used to reason about the type system in Section 4.

\subsection{Unification}

This section describes the unification algorithm which is a central component of the type inference algorithm. A substitution $S$ is called a unifier for the type expressions $\tau$ and $\tau^{\prime}$ if $S \tau=S \tau^{\prime}$. The following theorem is due to [Rob65].

Theorem 4 (Unification algorithm) There is an algorithm whose input is a pair of type expressions $\tau$ and $\tau^{\prime}$ such that either:

the algorithm succeeds with a substitution $U$ as its result and the unifiers of $\tau$ and $\tau^{\prime}$ are precisely those substitutions of the form $R U$ for any substitution $R$. The substitution $U$ is called a most general unifier for $\tau$ and $\tau^{\prime}$, and is denoted $m g u\left(\tau, \tau^{\prime}\right)$.

or the algorithm fails and there are no unifiers for $\tau$ and $\tau^{\prime}$.

In the following, we write $\tau \stackrel{U}{\sim} \tau^{\prime}$ for the assertion that the unification algorithm succeeds by finding a most general unifier $U$ for $\tau$ and $\tau^{\prime}$. 


\subsection{Type inference algorithm $\mathrm{W}$}

Following the presentation of [Rem89], we describe the type inference algorithm using the inference rules in Figure 5. These rules use typings of the form $P \mid T A \stackrel{\text { w }}{\vdash} M: \tau$ where $P$ is a set of predicates, $T$ is a substitution, $A$ is a type assignment, $M$ is a term and $\tau$ is a simple type expression. The typing rules can be interpreted as an attribute grammar in which $A$ are $M$ inherited attributes, while $P, T$ and $\tau$ are synthesised.

\begin{tabular}{|ccc|}
\hline & $\frac{\left(x: \forall \alpha_{i} . P \Rightarrow \tau\right) \in A}{\left[\beta_{i} / \alpha_{i}\right] P \mid A \stackrel{w}{\vdash} x:\left[\beta_{i} / \alpha_{i}\right] \tau}$ & $\beta_{i}$ new \\
$(\rightarrow E)^{\mathrm{w}}$ & $\frac{P|T A \stackrel{\mathrm{w}}{\vdash} M: \tau \quad Q| T^{\prime} T A \stackrel{\mathrm{w}}{\vdash} N: \tau^{\prime} \quad T^{\prime} \tau \stackrel{U}{\sim} \tau^{\prime} \rightarrow \alpha}{U\left(T^{\prime} P \cup Q\right) \mid U T^{\prime} T A \stackrel{\mathrm{w}}{\vdash} M N: U \alpha}$ & $\alpha$ new \\
$(\rightarrow I)^{\mathrm{w}}$ & $\frac{P \mid T\left(A_{x}, x: \alpha\right) \stackrel{\mathrm{w}}{\vdash} M: \tau}{P \mid T A \stackrel{\mathrm{w}}{\vdash} \lambda x \cdot M: T \alpha \rightarrow \tau}$ & $\alpha$ new \\
& $\frac{P\left|T A \stackrel{\mathrm{w}}{\vdash} M: \tau P^{\prime}\right| T^{\prime}\left(T A_{x}, x: \sigma\right) \stackrel{\mathrm{w}}{\vdash} N: \tau^{\prime}}{P^{\prime} \mid T^{\prime} T A \stackrel{\mathrm{w}}{\vdash}(\operatorname{let} x=M \text { in } N): \tau^{\prime}}$ & $\sigma=\operatorname{Gen}(T A, P \Rightarrow \tau)$
\end{tabular}

Figure 5: Type inference algorithm $\mathrm{W}$

The algorithm may also be described in a more conventional style by defining a function $W$ such that $P \mid T A \stackrel{w}{\vdash} M: \tau$ if and only if $W(A, M)$ succeeds with result $(P, T, \nu)$. One of the advantages of our choice of notation is that it highlights the the relationship between $W$ and the syntax-directed type system, as illustrated by the following theorem.

Theorem 5 If $P \mid T A \stackrel{\text { }}{\vdash} M: \tau$ then $P \mid T A \dot{\vdash} M: \tau$.

Combining this with the result of theorem 2 gives the following important corollary.

Corollary 1 (Soundness of $W$ ) If $P \mid T A \stackrel{w}{\vdash} M: \tau$ then $P \mid T A \vdash M: \tau$.

With the exception of $(l e t)^{\mathrm{w}}$, each of the rules in Figure 5 introduces 'new' variables; i.e. variables which do not appear in the hypotheses of the rule nor in any other distinct branches of the complete derivation. Note that it is always possible to choose type variables in this way because the set of type variables is assumed to be countably infinite. In the presence of new variables, it is convenient to work with a weaker form of equality on substitutions, writing $S \approx R$ to indicate that $S t=R t$ for all but a finite number of new variables $t$. In most cases, we can treat $S \approx R$ as $S=R$, since the only differences between the substitutions occur at variables which are not used elsewhere in the algorithm.

This notation enables us to give an accurate statement of the following result which shows that the typings obtained by $W$ are, in a precise sense, the most general derivable typings for a given term.

Theorem 6 Suppose that $P \mid S A \stackrel{\vdash}{\vdash} M: \tau$. Then $Q \mid T A \stackrel{w}{\vdash} M: \nu$ and there is a substitution $R$ such that $S \approx R T, \tau=R \nu$ and $P H R Q$. 
Combining the result of theorem 6 with that of theorem 3 we obtain a similar completeness result for $W$ with respect to the type system of Section 4 .

Corollary 2 Suppose that $P \mid S A \vdash M: \sigma$. Then $Q \mid T A \stackrel{\text { w }}{\vdash} M: \nu$ and there is a substitution $R$ such that $S \approx R T$ and $(P \mid \sigma) \leq R G e n(T A, Q \Rightarrow \nu)$.

\subsection{Principal type schemes}

The principal type scheme of a term corresponds to the most general derivable typing with respect to the ordering on type schemes under a given type assignment.

Definition $6 A$ principal type scheme for a term $M$ under a type assignment $A$ is a constrained type scheme $(P \mid \sigma)$ such that $P \mid A \vdash M: \sigma$, and $\left(P^{\prime} \mid \sigma^{\prime}\right) \leq(P \mid \sigma)$ whenever $P^{\prime} \mid A \vdash M: \sigma^{\prime}$.

The following result gives a sufficient condition for the existence of principal type schemes, by showing how they can be constructed from typings produced by $W$.

Corollary 3 Suppose that $M$ is a term, $A$ is a type assignment and $Q \mid T A \stackrel{\text { w }}{\vdash} M: \nu$ for some $Q, T$ and $\nu$. Then $\operatorname{Gen}(T A, Q \Rightarrow \nu)$ is a principal type scheme for $M$ under TA.

Combining this with corollary 2 gives a necessary condition for the existence of principal type schemes: a term is well-typed if and only if it has a principal type scheme which can be calculated using the type inference algorithm.

\section{Topics for further research}

\subsection{The coherence problem}

At this point, it is important to point out that the type systems described by the rules in the previous sections are not coherent (in the sense of [BCGS89]). In other words, it is possible to construct translations $P \mid A \vdash M \sim M_{1}^{\prime}: \sigma$ and $P \mid A \vdash M \sim M_{2}^{\prime}: \sigma$ in which the terms $M_{1}^{\prime}$ and $M_{2}^{\prime}$ are not equivalent, and hence the semantics of $M$ are not well-defined.

For an example in which the coherence problem arises, consider the term out (in $x$ ) under the evidence assignment $P=\{u: C$ Int $, v: C B o o l\}$ and the type assignment:

$$
A=\{x: \text { Int }, \text { in }: \forall a . C a \Rightarrow \text { Int } \rightarrow a, \text { out }: \forall a . C a \Rightarrow a \rightarrow \text { Int }\}
$$

for some unary predicate symbol $C$. Instantiating the quantified type variable in the type of in (and hence also in that of out) with the types $I n t$ and Bool leads to distinct derivations $P \mid A \vdash$ out (in $x$ ) : Int in which the corresponding translations, out $u$ (in $u x$ ) and out $v$ (in $v x$ ) are clearly not equal.

Note that the principal type scheme of out (in $x$ ) in this example is $\forall a . C a \Rightarrow I n t$ and that the type variable $a$ (the source of the lack of coherence in the derivations above) appears only in the predicate qualifying the type of the term, not in the type itself. Motivated by the functional programming language Haskell [HPJW91], we say that a type of the form $\forall \alpha_{i} . P \Rightarrow \tau$ is unambiguous 
if $T V(P) \subseteq T V(\tau)$. It is believed that the coherence problem described above can be avoided by prohibiting the use of terms with ambiguous principal type schemes and restricting type assignments to types containing only unambiguous type schemes. A similar result has been established in [Blo90] for the special case of [WB89]. We expect this result to generalise to the framework used in this paper.

\subsection{Eliminating evidence parameters}

Using translations as described in Section 3 , a term $M$ of type $\forall \alpha_{i} . P \Rightarrow \tau$ will be implemented by a term of the form $\lambda v_{1} \ldots \lambda v_{n} . M^{\prime}$, where $P=\left\{\pi_{1}, \ldots, \pi_{n}\right\}$ and each $v_{i}$ is an evidence variable for the corresponding predicate $\pi_{i}$. In the following sections we describe a number of situations in which the number of evidence parameters required can be reduced using a more sophisticated variant of the type inference algorithm.

\subsubsection{Minimisation/simplification}

The translation of a term whose type is qualified by a set of predicates $P$ requires one evidence abstraction for each element of $P$. Thus the number of evidence parameters that are required can be reduced by finding a smaller set of predicates $Q$, equivalent to $P$ in the sense that $P+Q$ and $Q$ H $P$ (and hence the type of the new term is equivalent to that of the original term). In this situation, we have a compromise between:

- Reducing the number of evidence parameters required.

- The cost of constructing evidence for $P$ from the evidence supplied for $Q$. The fact that this is possible is guaranteed by the assertion $Q H P$.

In general, the task of finding an optimal set of predicates with which to replace $P$ is likely to be intractable. One potentially useful approach would be to determine a minimal subset $Q \subseteq P$ such that $Q \Vdash P$. To see that this is likely to be a good choice, note that:

- $P H Q$, by monotonicity of $H$ and hence $Q$ is equivalent to $P$ as required.

- Since $Q \subseteq P$, the number of evidence abstractions required using $Q$ is less than or equal to the number required when using $P$.

- The construction of evidence for a predicate in $P$ using evidence for $Q$ is trivial for each predicate which is already in $Q$.

\subsubsection{Evidence parameters considered harmful}

The principal motivation for including the let construct in the term language was to enable the definition and use of polymorphic and overloaded values. In practice, the same construct is also used for a number of other purposes:

- To avoid repeated evaluation of a value which is used at a number of points in an expression. 
- To create cyclic data structures using recursive bindings [BW89].

- To enable the use of identifiers as abbreviations for the subexpressions of a large expression.

Note however that the addition of evidence parameters to the value defined in a let expression may mean that the evaluation of an overloaded term will not behave as intended. For example, if $M$ is a term of type $\forall a . C a \Rightarrow a \rightarrow$ Int then we have:

$$
\text { let } x=M 1 \text { in } x+x \leadsto \lambda e . \operatorname{let} x=\left(\lambda v \cdot M^{\prime} v 1\right) \text { in }(x e+x e)
$$

and the evaluation of $x e$ in the translation is no longer shared. There are a number of potential solutions to this problem. In the example above, one method would be to transform the translated term to:

$$
\left.\lambda e . \text { let } x=\left(\lambda v \cdot M^{\prime} v 1\right) \text { in (let } y=x e \text { in } y+y\right) .
$$

Another possibility would be the use of a monomorphism restriction such as that proposed for Haskell [HPJW91] which restricts the amount of overloading which can be used in particular syntactic forms of binding. In this case, the corresponding translation is likely to be:

$$
\lambda e \text {.let } x=M^{\prime} e 1 \text { in } x+x .
$$

\subsubsection{Constant and locally-constant overloading}

Consider the typing of local definitions in the type system of Section 4 using the rule:

$$
\frac{P|A \vdash M: \sigma \quad Q| A_{x}, x: \sigma \vdash N: \tau}{P \cup Q \mid A \vdash(\operatorname{let} x=M \text { in } N): \tau}
$$

Notice that this allows some of the predicates constraining the typing of $M$ (i.e. those in $P$ ) to be retained as a constraint on the environment in the conclusion of the rule rather than being included in the type scheme $\sigma$. However, in the corresponding rule (let $)^{3}$ for the syntax-directed system, all of the predicates constraining the typing of $M$ are included in the type $\operatorname{Gen}(A, P \Rightarrow \tau)$ that is inferred for $M$ :

$$
\frac{P\left|A \stackrel{\leftrightarrow}{\vdash} M: \tau \quad P^{\prime}\right| A_{x}, x: \operatorname{Gen}(A, P \Rightarrow \tau) \dot{\vdash} N: \tau^{\prime}}{P^{\prime} \mid A \dot{\vdash}(\operatorname{let} x=M \text { in } N): \tau^{\prime}}
$$

As a consequence, evidence parameters are needed for all of the predicates in $P$, even if the evidence values used for some of these parameters are the same for each occurrence of $x$ in $N$. In particular, this includes constant evidence (corresponding to predicates with no free type variables) and locallyconstant evidence (corresponding to predicates, each of whose free variables also appears free in A).

From the relationship between the type inference algorithm $W$ and the syntax-directed system, it follows that $W$ has the same behaviour; indeed, this is essential to ensure that $W$ calculates principal types: If $x \notin F V(N)$ then none of the environment constraints described by $P$ need be reflected by the constraints on the complete expression in $P^{\prime}$.

However, if $x \in F V(N)$, it is possible to find a set $F \subseteq P$ such that $P^{\prime} H F$ and hence the type scheme assigned to $x$ can be replaced by $\operatorname{Gen}(A,(P \backslash F) \Rightarrow \tau)$, potentially decreasing the number 
of evidence parameters required by $x$. To see this, suppose that $\operatorname{Gen}(A, P \Rightarrow \tau)=\left(\forall \alpha_{i} . P \Rightarrow \tau\right)$. A straightforward induction, based on the hypothesis that $x \in F V(N)$, shows that $P^{\prime} \mathbb{H}\left[\tau_{i} / \alpha_{i}\right] P$ for some types $\tau_{i}$. If we now define:

$$
F P(A, P)=\{(v: \pi) \in P \mid T V(\pi) \subseteq T V(A)\}
$$

then $F=F P(A, P)$ is the largest subset of $P$ which is guaranteed to be unchanged by the substitution $\left[\tau_{i} / \alpha_{i}\right]$. These observations suggest that $(l e t)^{s}$ could be replaced by the two rules:

- In the case where $x \notin F V(N)$ :

$$
\frac{P\left|A \vdash M: \tau \quad P^{\prime}\right| A \stackrel{\bullet}{\vdash} N: \tau^{\prime}}{P^{\prime} \mid A \dot{\vdash}(\text { let } x=M \text { in } N): \tau^{\prime}}(\text { let })_{f}^{\circ}
$$

The typing judgement involving $M$ serves only to preserve to property that all subterms of a well-typed term are also well-typed.

- In the case where $x \in F V(N)$ :

$$
\frac{P\left|A \dot{\vdash} M: \tau \quad P^{\prime}\right| A_{x}, x: \operatorname{Gen}(A, P \backslash F \Rightarrow \tau) \dot{\vdash} N: \tau^{\prime} \quad P^{\prime} \Vdash F}{P^{\prime} \mid A \dot{\vdash}(\text { let } x=M \text { in } N): \tau^{\prime}}(\text { let })_{b}
$$

where $F=F P(A, P)$.

Whilst these rules retain the syntax-directed character necessary for use in a type inference algorithm, they are not suitable for typing top-level definitions (such as those in Haskell or ML) which are treated as let expressions in which the scope of the defined variable is not fully determined at compile-time. A more realistic approach would be to use just $(l e t)_{b}{ }^{\circ}$ in place of $(l e t)^{s}$, with the understanding that type schemes inferred by $W$ are only guaranteed to be principal in the case where $x \in F V(N)$ for all subterms of the form let $x=M$ in $N$ in the term whose type is being inferred. Justification for this approach is as follows:

- For a top-level declaration of the identifier $x$, we can take the scope of the declaration to be the set of all terms which might reasonably be evaluated in the scope of such a declaration, which of course includes the term $x$.

- For let expressions in which the scope of the defined variable is known, the local definition in an expression of the form let $x=M$ in $N$ is redundant, and the expression is semantically equivalent to $N$. However, expressions of this form are sometimes used in implicitly typed languages to force a less general type than might otherwise be obtained by the type inference mechanism. For example, if $(==)$ is an integer equality function and 0 is an integer constant, then $\lambda x$.let $y=(x==0)$ in $x$ has principal type scheme Int $\rightarrow$ Int, whereas the principal type scheme for $\lambda x . x$ is $\forall a . a \rightarrow a$. Such ad-hoc 'coding-tricks' become unnecessary if the term language is extended to allow explicit type declarations.

In a practical implementation, it would be useful to arrange for suitable diagnostic messages to be generated whenever an expression of the form let $x=M$ in $N$ with $x \notin F V(N)$ is encountered; this would serve as a warning to the programmer that the principal type property may be lost (in addition to catching other potential program errors). 


\subsection{The use of subsumption}

The typing rules in Figure 1 are only suitable for reasoning about systems with explicit coercions. For example, if $I n t \subseteq$ Real, then we can use an addition function:

$$
a d d:: \forall a . a \subseteq R e a l \Rightarrow a \rightarrow a \rightarrow \text { Real }
$$

to add two integers together, obtaining a real number as the result. More sophisticated systems, such as those in [Mit84, FM89], cannot be described without adding a form of the rule of subsumption:

$$
\frac{P \mid A \vdash M: \tau^{\prime} \quad P \Vdash \tau^{\prime} \subseteq \tau}{P \mid A \vdash M: \tau}
$$

Each use of this rule corresponds to an implicit coercion; the addition of two integers to obtain a real result can be described without explicit overloading using a function:

$$
\text { add : Real } \rightarrow \text { Real } \rightarrow \text { Real }
$$

with two implicit coercions from Int to Real. As a further example, in the framework of Section 2, the polymorphic identity function $\lambda t . \lambda x: t . x$ can be treated as having type $\forall a . \forall b . a \subseteq b \Rightarrow a \rightarrow b$ and hence acts as a generic coercion function.

No attempt has been made to deal with systems including the rule of subsumption in the development of the type inference algorithm in Section 6, which is therefore only suitable for languages using explicit coercions. The results of [FM89] and [Smi91] are likely to be particularly useful in extending the present system to support the use of implicit coercions.

\subsection{Other issues}

In addition to the problems already mentioned, we list the following topics as areas for further work.

- Semantic issues. The correspondence between typing derivations and translations provides a simple semantic interpretation for overloaded terms in a language without built-in support for overloading. By demonstrating that any translation of a well-typed term is itself well-typed, we can extend Milner's result for the ML type system that "well-typed programs do not go wrong" to the system described in Section 4.

- Decidability and satisfiability. It has recently been shown [VS91] that, using a definition of well-typing that takes account of the satisfiability of predicate sets, the task of determining whether a given expression is well-typed in an unrestricted version of [WB89] is undecidable. Clearly this is unacceptable for any practical application of such type systems. In the framework used in this paper, decidability of type checking is completely determined by the properties of $H$ and it remains an interesting problem to investigate what restrictions on the definition of $H$ are needed to guarantee decidability.

Further refinements are likely to be suggested by the development, currently in progress, of an implementation of qualified types, based on the ideas described in this paper [Jon92]. 


\section{Acknowledgements}

I am very grateful for conversations with members of the Department of Computing Science, Glasgow whose suggestions prompted me to investigate the ideas presented in this paper, and in particular to Phil Wadler for his comments on several earlier versions of this paper.

A number of features of this paper were motivated by the functional programming language Haskell [HPJW91]. The notation $\pi \Rightarrow \sigma$ for qualified types is modelled on the syntax of Haskell predicated types and the concept of evidence is a generalisation of Haskell dictionaries. The static semantics described in [PJW90] uses similar methods to those presented here, introducing explicit dictionary abstraction and application to make overloading explicit.

The Science and Engineering Council of Great Britain provided financial support for this work.

\section{References}

[BCGS89] V. Breazu-Tannen, T. Coquand, C. A. Gunter and A. Scedrov. Inheritance and coercion. Proceedings of the fourth annual symposium on logic in computer science, 1989.

[Blo90] Stephen Blott. An approach to overloading with polymorphism. Ph.D. thesis, Department of computing science, University of Glasgow, 1990 (in preparation).

[BW89] Richard Bird and Philip Wadler. Introduction to functional programming. Prentice Hall International, 1989.

[CDK86] Dominique Clément, Joëlle Despeyroux, Thierry Despeyroux and Gilles Kahn. A simple applicative language: Mini-ML. ACM symposium on LISP and functional programming, 1986.

[CM90] Luca Cardelli and John Mitchell. Operations on records. Proceedings of the Fifth International Conference on Mathematical Foundations of Programming Language Semantics. Lecture notes in computer science 442, Springer Verlag, 1990.

[Dam85] Luis Damas. Type assignment in programming languages. PhD thesis, University of Edinburgh, CST-33-85, 1985.

[DM82] Luis Damas and Robin Milner. Principal type schemes for functional programs. Proceedings of the 8th annual ACM symposium on Principles of Programming languages, Albuquerque, New Mexico, January 1982.

[FM89] You-Chin Fuh and Prateek Mishra. Polymorphic subtype inference: Closing the theorypractice gap. Lecture notes in computer science 352, Springer Verlag, 1990.

[HP90] Robert W. Harper and Benjamin C. Pierce. Extensible records without subsumption. Technical report CMU-CS-90-102, Carnegie Mellon University, School of computer science, February 1990. 
[HPJW91] Paul Hudak, Simon Peyton Jones and Philip Wadler (editors). Report on the programming language Haskell, a non-strict purely functional language (Version 1.1). Technical Report YALEU/DCS/RR777, Yale University, Department of Computer Science, August 1991.

[Jon91a] Mark P. Jones. Towards a theory of qualified types. Technical report PRG-TR-6-91, Programming Research Group, Oxford University Computing Laboratory, April 1991.

[Jon91b] Mark P. Jones. Type inference for qualified types. Technical report PRG-TR-10-91, Programming Research Group, Oxford University Computing Laboratory, June 1991.

[Jon92] Mark P. Jones Practical issues in the implementation of qualified types. Forthcoming technical report, Oxford University Computing Laboratory, 1992.

[Mil78] Robin Milner. A theory of type polymorphism in programming. Journal of Computer and System Sciences, 17, 3, 1978.

[Mit84] John C. Mitchell. Coercion and type inference (summary). Proceedings of the 11th annual ACM symposium on Principles of Programming Languages, 1984.

[Mit90] John C. Mitchell A type-inference approach to reduction properties and semantics of polymorphic expressions. Logical Foundations of Functional Programming, Gérard Huet (ed.), Addison Wesley, 1990.

[PJW90] Simon L. Peyton Jones and Philip Wadler A static semantics for haskell (draft). Department of Computing Science, University of Glasgow, August 1990.

[Rem89] Didier Rémy. Typechecking records and variants in a natural extension of ML. Proceedings of the 16th annual ACM symposium on Principles of Programming Languages, Austin, Texas, January 1989.

[Rob65] J. A. Robinson. A machine-oriented logic based on the resolution principle. Journal of the Association for Computing Machinery, 12, 1965.

[Smi91] Geoffrey Smith. Polymorphic type inference for languages with overloading and subtyping. Ph.D. thesis, Department of Computer Science, Cornell University, August 1991.

[VS91] Dennis Volpano and Geoffrey Smith. On the complexity of ML typability with overloading. Proceedings of the 5th ACM conference on Functional Programming Languages and Computer Architecture. Lecture notes in computer science 523, Springer Verlag, 1991.

[WB89] Philip Wadler and Stephen Blott. How to make ad-hoc polymorphism less ad-hoc. Proceedings of the 16th annual ACM symposium on Principles of Programming Languages, Austin, Texas, January 1989. 